Sharif University of Technology
Scientia Iranica
SCIENTIA

\title{
Effects of particle shape and size distribution on particle size-dependent flow strengthening in metal matrix composites
}

\author{
S. Soleymani Shishvan* and A.-H. Asghari \\ Faculty of Civil Engineering, University of Tabriz, Tabriz, P.O. Box 51666-16471, Iran.
}

Received 8 August 2015; received in revised form 2 May 2016; accepted 19 July 2016

\section{KEYWORDS}

Particle-reinforced composites;

Mechanical properties; Plastic deformation;

Modeling.

\begin{abstract}
Effects of particle size on metal matrix composites are studied within the Continuum theory of Mechanism-based Strain Gradient (CMSG) plasticity. This theory has been quite successful in predicting the size-dependent plastic behavior in a wide variety of problems. Two-dimensional (plane-strain) analyses carried out on the composite unit cell models with multi-particles of circular shape show that the flow stress of the composites increases by decreasing particle size with high sensitivity to small particle size. The numerical results are in good agreement with experimental data. Subsequently, the effects of particle shape, orientation, and size distributions on the behavior of composites are investigated. Analyses are carried out on the composites containing squared, rectangular, and elliptical (with aspect ratio of four) particles of various orientations with respect to the loading direction (i.e., vertical, horizontal, and 45 degree inclined directions). The stress inhomogeneity in the matrix, the overall stress-strain curve, and the maximum principle stress in the particles of composites with non-circular particles are investigated and compared with those obtained for the composites containing circular particles. The effects of particle size distribution on the behavior of composites are also addressed.
\end{abstract}

(C) 2017 Sharif University of Technology. All rights reserved.

\section{Introduction}

The mechanical properties of particle reinforced metal matrix composites have received considerable attention in the last few decades due to their intensive applications in various areas from civil constructions to aerospace industries. The overall behavior of composites is mainly controlled by particles through both volume fraction of particles and particle sizes. Although the former contribution is now well understood, the latter one in combination with the particle shapes, orientations, positions, and size distributions is still under investigation by researchers.

*. Corresponding author. Tel.: +984133392396 E-mail address: sshishvan@tabrizu.ac.ir (S. Soleymani Shishvan)
Experimentally observed particle size effects [15] cannot be generally predicted by the classical plasticity theories, since their constitutive laws possess no internal material lengths. However, consideration of quench hardening may assist the classical plasticity in predicting particle size effects [6] where the particle size is greater than $\sim 25 \mu \mathrm{m}$. The literature in this field certainly confirms that a theory of Strain Gradient Plasticity (SGP) is needed to investigate the effects of particle size. It is, however, noted that other methods based on essential features of dislocation plasticity have also been successful in doing so $[7,8]$.

Xue et al. [9] used the theory of Mechanism-based Strain Gradient (MSG) plasticity [10] to predict the particle size effects. They employed a single-particle axisymmetric unit cell model [11] of the $\mathrm{SiC} / \mathrm{Al}$ composite, and their results were in good agreement with 
the experiments of [1]. Qu et al. [12] analyzed the same model within the Conventional theory of Mechanismbased Strain Gradient (CMSG) plasticity [13], the loworder form of the MSG plasticity. They not only extended the theory to incorporate quench hardening, but also used the cohesive zone model to account for interfacial debonding; indeed, their results were in excellent agreement with experiments of [1]. Shao et al. [14] also analyzed this model within the Taylorbased Nonlocal Theory (TNT) of plasticity [15] where the dislocation punched zone [16] was also defined around the particle to account for quenching. While numerical results well predicted the particle size effects, it was the incorporation of the cohesive zone model that improved the results in confrontation with experiments of [1]. On the other hand, Yan et al. [5] carried out analyses on multi-particle (plane-strain) unit cell models of the $\mathrm{SiC} / \mathrm{Al}$ composites within the TNT plasticity [15]. They considered circular particles of four average sizes $1,5,20$, and $56 \mu \mathrm{m}$ that were randomly distributed in the matrix. Their numerical results were in qualitative agreement only with their own experimental data [5]. In another remarkable study, Fredriksson et al. [17] modeled composites with rectangular particles where they introduced a general finite element framework for the higher order SGP theory of Gudmundson [18], and showed that strengthening can be captured due to particle size, but strengthening cannot be captured due to particle shape.

Alternatively, Shishvan and Asghari [19] have recently studied the plastic behavior of particle reinforced composites by the continuum theory of stress gradient plasticity $(\sigma$ GP) $[20,21]$. This theory has been quite successful in predicting the size-dependent behavior in micro-torsion and -bending [20-22]. Their results reveal that the experimental observations of particle size effect can be explained in terms of a stress gradient plasticity theory with one material length variable, which depends on the matrix microstructure. However, the $\sigma$ GP theory is not considered here, and we only use the CMSG theory of plasticity which is mainly justifiable with the scope of the present study and computationally less expensive than the MSG, TNT, and $\sigma$ GP theories.

In the present paper, we apply the Finite Element Method (FEM) by incorporating the CMSG theory of plasticity to investigate the effects of particle position, shape, orientation, and size distribution on the size-dependent flow strengthening of the $\mathrm{SiC} / \mathrm{Al}$ composites. It is noted that the classical plasticity theories have already been employed to study the effects of particle shape [23,24] as well as the particle orientation [4] on the behavior of composites, even though the particle size-dependent behavior has not been the main concern in such studies. Here, the particle size effects on the behavior of metal matrix composites are investigated in combination with the particle features, which have not been considered up to now.

It is also noteworthy that all the above mentioned models are two-dimensional; however, there exist threedimensional models of composites within the classical plasticity theories [6]. Here, we use two-dimensional models for our investigations.

The paper is organized as follows. In Section 2, the CMSG theory of plasticity is recapitulated. Section 3 is devoted to describe the finite element model and material properties used in simulations. Section 4 presents numerical results that are compared with experiments and the effects of particle features including shape, orientation, and size distributions on the particle size-dependent flow strengthening of composites are appropriately addressed.

\section{CMSG theory of plasticity}

CMSG was formulated by Huang et al. [13] based on the Taylor dislocation model [25], namely $\tau=\alpha G b \sqrt{\rho}$ with $\tau$ the stress, $G$ the shear modulus, $\alpha$ as an empirical constant, $b$ the length of Burgers vector, and $\rho$ the total dislocation density that comprises statistically stored dislocation density $\left(\rho_{S}\right)$ and geometrically necessary dislocation density $\left(\rho_{G}\right)$, i.e. $\rho=\rho_{S}+\rho_{G}$. According to the relation between tensile and shear flow stresses, i.e. $\sigma_{\text {flow }}=M \tau$ with $M$ the Taylor factor, the (tensile) flow stress in CMSG can be expressed as [13]:

$$
\sigma_{\text {flow }}=\sigma_{\text {ref }} \sqrt{\left(f\left(\varepsilon^{p}\right)\right)^{2}+l \eta^{p}}
$$

with the material length scale:

$$
l=M^{2} \bar{r} \alpha^{2}\left(G / \sigma_{\mathrm{ref}}\right)^{2} b,
$$

where $\sigma_{\text {ref }}$ is reference stress, $f$ is non-dimensional function determined from the uniaxial tensile test, and $\bar{r}$ is Nye factor [26]. For FCC metals: $\bar{r}=1.90$ and $M=3.06$ [12]. Effective plastic strain gradient $\eta^{p}$ is given by $[12,13]$ :

$$
\begin{aligned}
& \eta^{p}=\int \dot{\eta}^{p} d t=\int \sqrt{\frac{1}{4} \dot{\eta}_{i j k}^{p} \dot{\eta}_{i j k}^{p}} d t, \\
& \dot{\eta}_{i j k}^{p}=\dot{\varepsilon}_{i k, j}^{p}+\dot{\varepsilon}_{j k, i}^{p}-\dot{\varepsilon}_{i j, k}^{p},
\end{aligned}
$$

where $\dot{\varepsilon}_{i j}^{p}$ is the plastic strain rate tensor.

The constitutive equation in the CMSG plasticity theory is formally identical to the classical plasticity theory which is written as:

$$
\dot{\varepsilon}_{i j}=\left(\frac{1}{2 G} \dot{s}_{i j}+\frac{\dot{\sigma}_{k k}}{9 K} \delta_{i j}\right)+\frac{3 \dot{\bar{\varepsilon}}^{p}}{2 \sigma_{e}} s_{i j}
$$


where $K$ is the bulk modulus and $s_{i j}\left(=\sigma_{i j}-\sigma_{k k} \delta_{i j} / 3\right)$ is the deviatoric stress. The effective stress is defined by $\sigma_{e}=\sqrt{3 s_{i j} s_{i j} / 2}$ as in conventional rate-independent plasticity theories. However, equivalent plastic strain rate $\dot{\bar{\varepsilon}}^{p}$ is determined by the viscoplastic formulation introduced by Kok et al. [27] and adopted in CMSG [13], i.e.:

$$
\dot{\vec{\varepsilon}}^{p}=\dot{\varepsilon}\left(\frac{\sigma_{e}}{\sigma_{\text {flow }}}\right)^{m},
$$

where $m$ is the rate-sensitivity exponent and $\dot{\varepsilon}=$ $\sqrt{2 \dot{e}_{i j} \dot{e}_{i j} / 3}$ is the equivalent strain rate with $\dot{e}_{i j}(=$ $\left.\dot{\varepsilon}_{i j}-\dot{\varepsilon}_{k k} \delta_{i j} / 3\right)$ as the deviatoric strain rate. For large values of $m$, a rate-independent plasticity with flow stress, $\sigma_{\text {flow }}=\sigma_{\text {ref }} f\left(\bar{\varepsilon}^{p}\right)$, is achieved [13]. We adopt, in this paper, $m=20$ as in $[12,13]$.

Eq. (5) can be inverted and expressed as:

$$
\begin{aligned}
\dot{\sigma}_{i j}= & K \dot{\varepsilon}_{k k} \delta_{i j} \\
& +2 G\left\{\dot{e}_{i j}-\frac{3 \dot{\varepsilon}}{2 \sigma_{e}}\left[\frac{\sigma_{e}}{\sigma_{\text {ref }} \sqrt{\left(f\left(\varepsilon^{p}\right)\right)^{2}+l \eta^{p}}}\right]^{m} s_{i j}\right\},
\end{aligned}
$$

where Eq. (6) is used for $\dot{\bar{\varepsilon}}^{p}$ with $\sigma_{\text {flow }}$ substituted from Eq. (1). This equation represents the constitutive relation of the CMSG theory of plasticity. This constitutive relation accounts for the size effect which involves only the conventional stress and strain. Therefore, the CMSG does not require new finite elements, and it is straightforwardly implemented in the finite element program by the use of conventional finite elements. Here, we have implemented it in the ABAQUS finite element program via its USER-MATERIAL subroutine UMAT [28]. The only effort beyond that in classical plasticity is the evaluation of effective plastic strain gradient $\eta^{p}$ within UMAT. According to well-known methods in conventional plasticity theories, a constitutive integration algorithm is implemented and also the material consistent Jacobian is expressed in a closedform. It is noted that the equilibrium equations are identical to those in classical continuum theories.

\section{Finite element model and material descriptions}

We apply CMSG within a finite element analysis to the SiCp/Al composites studied experimentally by Yan et al. [5]. The composites contain $20 \%$ volume fraction of $\mathrm{SiC}$ particles that are distributed uniformly in the Al matrix and have four average sizes: 1, 5, 20, and $56 \mu \mathrm{m}$. As shown in Figure 1(a), a multi-particle unit cell model under plane-strain conditions is designed to investigate deformation of the composites. In this figure, particles are circular shape, but it should be noted that other shapes of particles are also used in

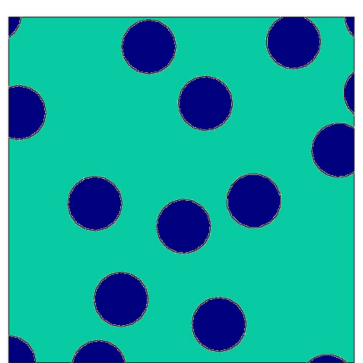

(a)

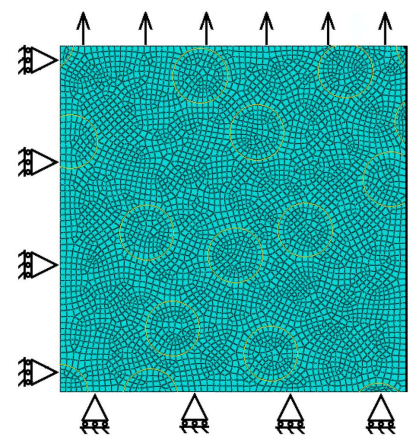

(b)
Figure 1. (a) A unit cell model of composite with circular particles. (b) The corresponding finite element model used in the analyses showing the mesh and boundary conditions.

the simulations (cf. Section 4.2). The particles are randomly positioned in the unit cell, and to reduce the statistical effects, various realizations of particle locations are considered for each case. The corresponding stress-strain curves are averaged and the scatter caused by the different realizations will be shown as an error bar.

The corresponding finite element model is shown in Figure 1(b) where the boundary conditions for the problem are also depicted as:

(i) $u_{x}=0$ at the left edge;

(ii) $u_{y}=0$ at the bottom edge;

(iii) All nodes at the right edge have common unknown $u_{x}$

(iv) Displacement $u_{y}$ is imposed on the top edge through which the average true strain in the tensile direction of the composites in the unit cell is calculated by $\varepsilon_{t}=\ln \left(1+u_{y} / L\right)$ with $L$ as the edge length of the unit cell.

These boundary conditions are adopted from [5], even though they are somehow over-constraining. A unit cell model with periodic characteristics and periodic boundary conditions can be used to improve the numerical model. A finite element mesh of biquadratic quadrilateral elements is used where the mesh is adequately refined to obtain a satisfactory computational accuracy. The nodes of the FE mesh for the particles and matrix are coinciding at the interfaces.

The tensile stress-strain data for unreinforced pure $\mathrm{Al}$ can be represented by:

$$
\sigma=\sigma_{Y}\left(1+\varepsilon^{p} / \varepsilon_{0}\right)^{N}=\sigma_{\text {ref }}\left(\varepsilon_{0}+\varepsilon^{p}\right)^{N},
$$

where $\sigma_{\text {ref }}=\sigma_{Y} / \varepsilon_{0}^{N}$. Parameters $\sigma_{Y}, \varepsilon_{0}$, and $N$ are chosen to fit experimental data with pure $\mathrm{Al}$ given in [5]. They are: $\sigma_{Y}=63 \mathrm{MPa}, \varepsilon_{0}=45 \times 10^{-6}$, and $N=0.105$, so $\sigma_{\text {ref }}=180.2 \mathrm{MPa}$. The intrinsic material length scale of $\mathrm{Al}$ is calculated from Eq. (2) 
as $l=9.77 \mu \mathrm{m}$. Elasticity for pure $\mathrm{Al}$ is taken to be isotropic with Young's modulus, $E=70 \mathrm{GPa}$, and Poisson's ratio, $\nu=0.33$, so that $G=26.3 \mathrm{GPa}$. The $\mathrm{SiC}$ particle is linear elastic and isotropic with $E_{\mathrm{SiC}}=450 \mathrm{GPa}$ and $\nu_{\mathrm{SiC}}=0.17$.

\section{Numerical results and discussion}

\subsection{Particle size effects}

Predictions of tensile stress-strain curves of composites with circular particles obtained using CMSG are plotted in Figure 2, which are averaged from four realizations and compared with the experimental data reported in [5]. It is seen that the CMSG theory can predict particle size-dependent flow strengthening in the composites so that the flow stress of the composites increases by decreasing particle size with high sensitivity to small particle size. The stress-strain curves of the CMSG agree well with the experimental data at small plastic strains, say $\sim 0.4 \%$, for all the composites. However, they tend to overestimate the hardening rate somewhat. Possible reasons for this can be mainly attributed to the fact that the present model does not account for contributions of particle cracking and interface debonding during deformation. Inclusion of these two features within a multi-particle model produces some numerical complexities; therefore, we will continue to investigate the effects of particle shape and size distribution on the behavior of composites by excluding such features in the next sections.

Contour plots of distribution of von Mises effective stress in the matrix of composites at the average axial strain $1 \%$ are shown in Figure 3. The upper contour limit (i.e., red color) for each case is adjusted with the average total stress in the composite (cf. stress-strain curves in Figure 2). The mismatch

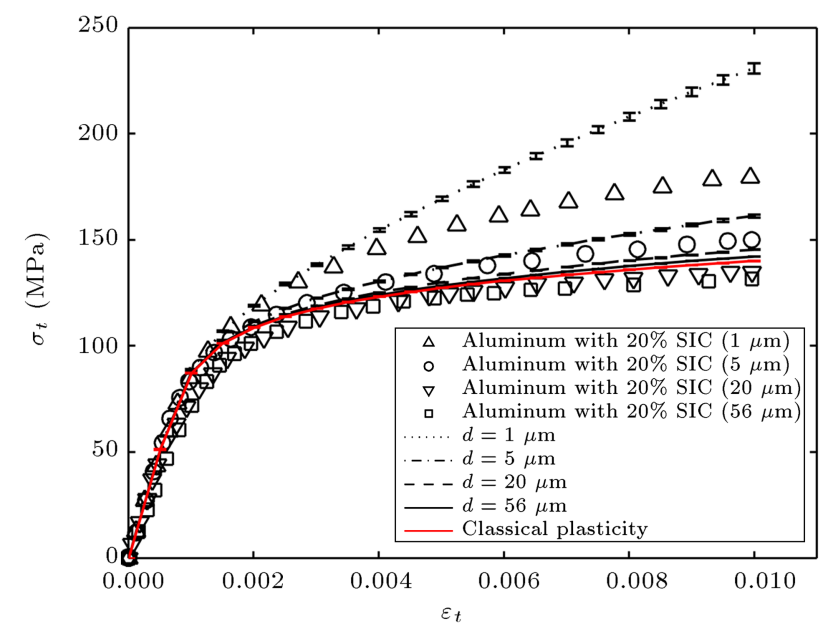

Figure 2. Predicted true stress-strain curves for the $20 \%$ $\mathrm{SiCp} / \mathrm{Al}$ composites with various sizes of circular particles. For comparison purposes, the experimental data from Yan et al. [5] are also plotted with symbols.

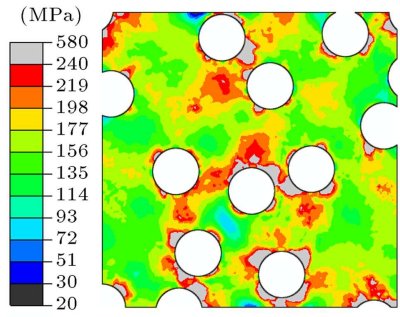

(a)

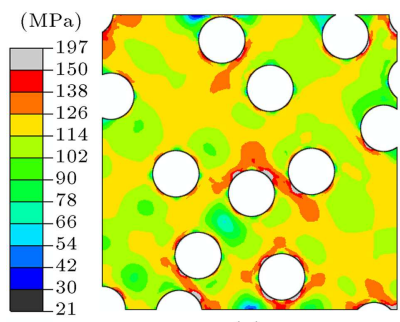

(c)

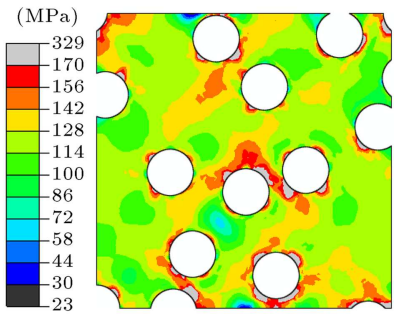

(b)

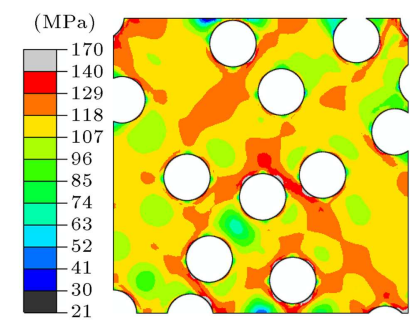

(d)
Figure 3. Contours of von Mises effective stress in the matrix at the average axial strain $1 \%$ for particle sizes: (a) $1 \mu \mathrm{m}$, (b) $5 \mu \mathrm{m}$, (c) $20 \mu \mathrm{m}$, and (d) $56 \mu \mathrm{m}$.

of elastic module between the particles and matrix originates the stress inhomogeneity in the matrix. Thus, plasticity would first occur in the region between neighboring particles with the largest stress of the matrix. Inhomogeneous plastic deformations in the matrix would then raise plastic strain gradients that determine the flow stress in the matrix within the CMSG theory of plasticity. It is seen in Figure 3 that by decreasing particle size, the stress distribution in the matrix becomes more and more inhomogeneous with a higher maximum value, i.e. the main origin of the observed particle size strengthening is the dislocation strengthening mechanism rather than the load transfer strengthening mechanism.

\subsection{Particle shape and orientation effects}

In order to investigate the effects of particle shape, we model composites with particles of squared, rectangular, and elliptical shape. Furthermore, different orientations of these non-circular particles are considered as shown in Figure 4. While the vertical (horizontal) particles indicate that the longer dimension of the particles is along (perpendicular to) the loading direction, the inclined particles have 45-degree angle of inclination with respect to the loading direction. It is noted that the dimensions of a non-circular particle are determined by equating its area with the circular one, so that the diameter of circular particle will be referred to as the size of a non-circular particle. The aspect ratio of rectangular and elliptical particles is chosen as four. As seen in Figure 4, the number and location of different shaped particles are to be kept unchanged as much as possible throughout entire models for each realization. Figure 4 shows contours of von Mises effective stress distributions in the matrix 


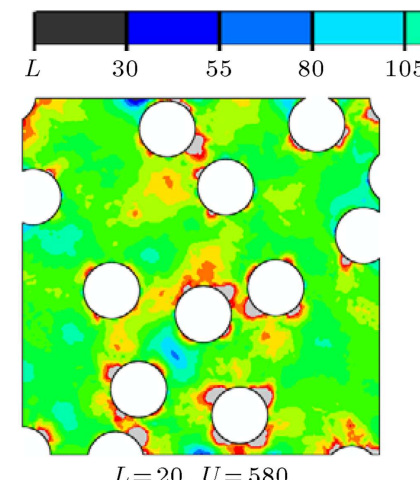

$=20, U=580$

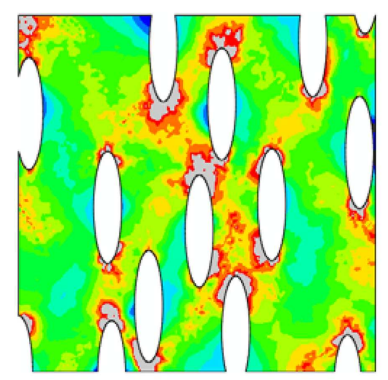

$L=16, U=614$

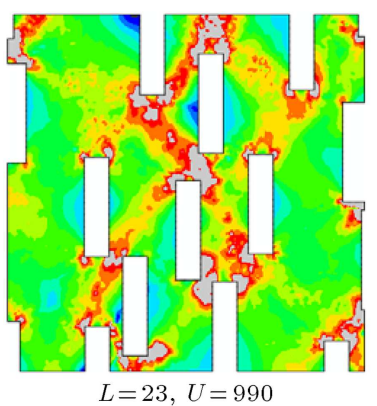

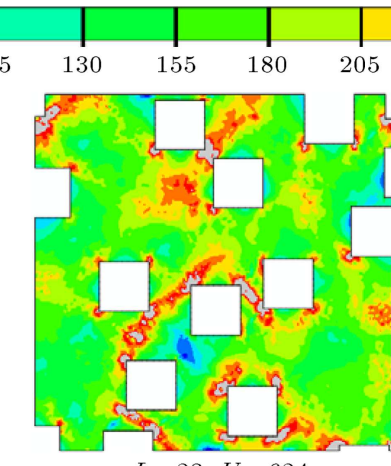

$L=23, U=624$

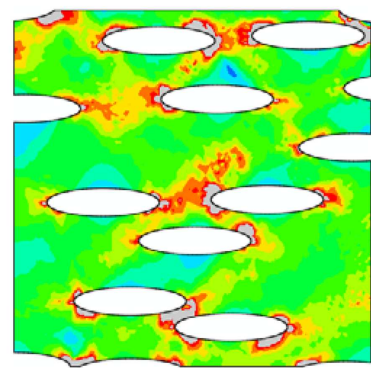

$L=30, U=549$

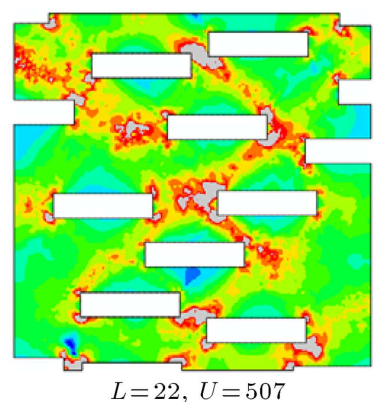

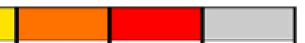

$230 \quad 255 \quad 280 \quad U(\mathrm{MPa})$
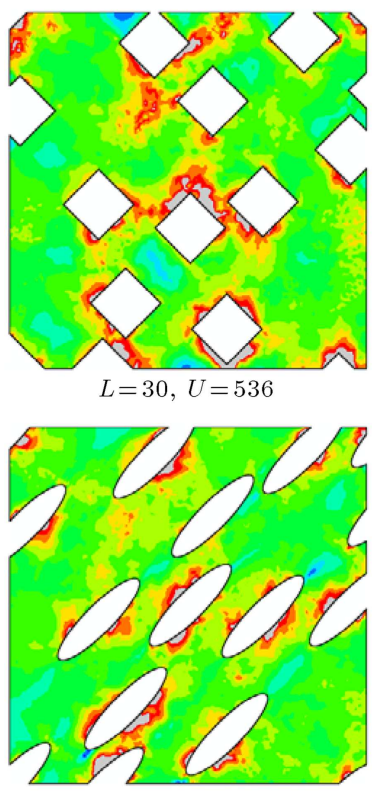

$L=27, U=448$

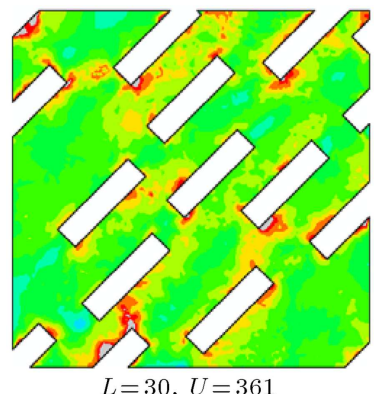

Figure 4. Contour plots of von Mises effective stress in the matrix at the average axial strain $1 \%$ for the composites of $1 \mu \mathrm{m}$ particle.

for these composites of $1 \mu \mathrm{m}$ particle size, where shape and orientation effects of particles are qualitatively demonstrated. It is clearly seen that the stress inhomogeneity in the matrix depends strongly on the shape and orientation of particles.

Tensile stress-strain curves obtained for these composites are shown in Figure 5 where the curves are averaged from four realizations and the legend is sorted by flow stress in descending order. It is observed that size effects predicted within CMSG theory depend on the shape and orientation of particles in the composites with quite high sensitivity to small particle size. This implies that for the small particle sizes, the shape and orientation of particles have significant effect on the dislocation strengthening mechanism. For the large particle sizes, the strengthening mechanism is the load transferring; thus, the shape and orientation of particles have a mild effect. It is also seen that the aspect ratio of particle dimensions plays an important role. The flow stress of composites with inclined (rectangular/elliptical) particles of $1 \mu \mathrm{m}$ size is much lower than those with vertical and horizontal particles. A mild effect of corner angles of particles on flow stress is predicted, even though the corner angles strongly influence the stress inhomogeneity in the matrix, as seen in Figure 4.

Although particles are assumed to be elastic in the present models, particle fracture is important as it plays a crucial role in the overall failure of composites. Figure 6 shows the distribution of the maximum principle stresses in the particles of $1 \mu \mathrm{m}$ size that can be used to predict which particles will fracture as well as to investigate how particle shape and orientation will affect particle fracture. Before proceeding with the discussion of the results, it is important to note that the highest values of the maximum principle stresses occur at particles that cross the boundaries. The following discussion excludes these boundary particles as they are affected by the constraints used for the boundaries of the model.

Figure 6 shows that particles with higher aspect ratio that are vertical have higher maximum principle stress than that of the circular particles. Moreover, 


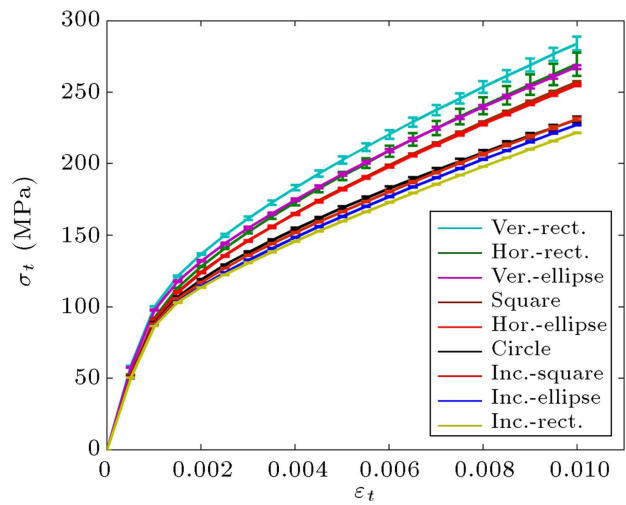

(a)

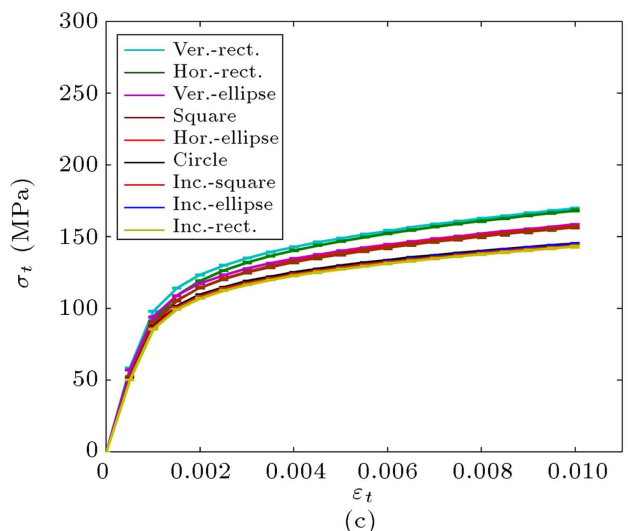

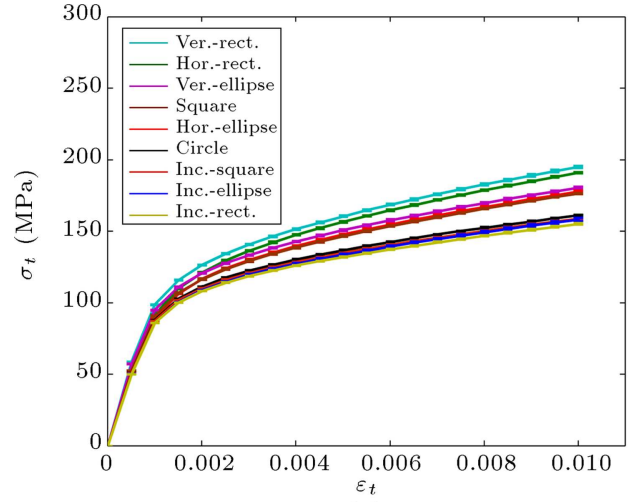

(b)

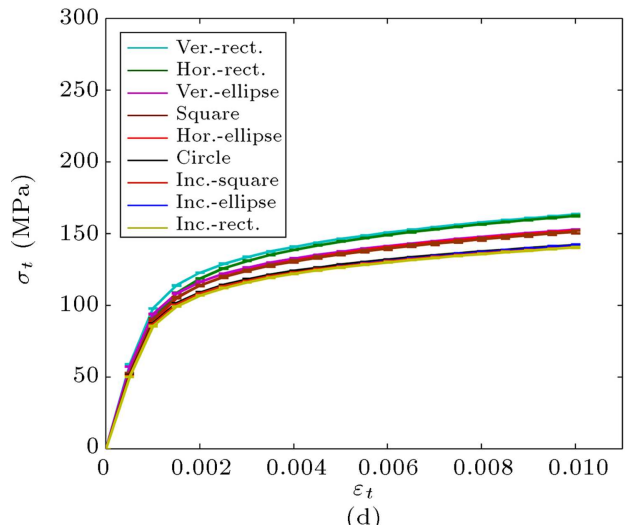

(d)

Figure 5. Predicted true stress-strain curves of the composites with various particle shapes and orientations of particle sizes: (a) $1 \mu \mathrm{m}$, (b) $5 \mu \mathrm{m}$, (c) $20 \mu \mathrm{m}$, and (d) $56 \mu \mathrm{m}$.

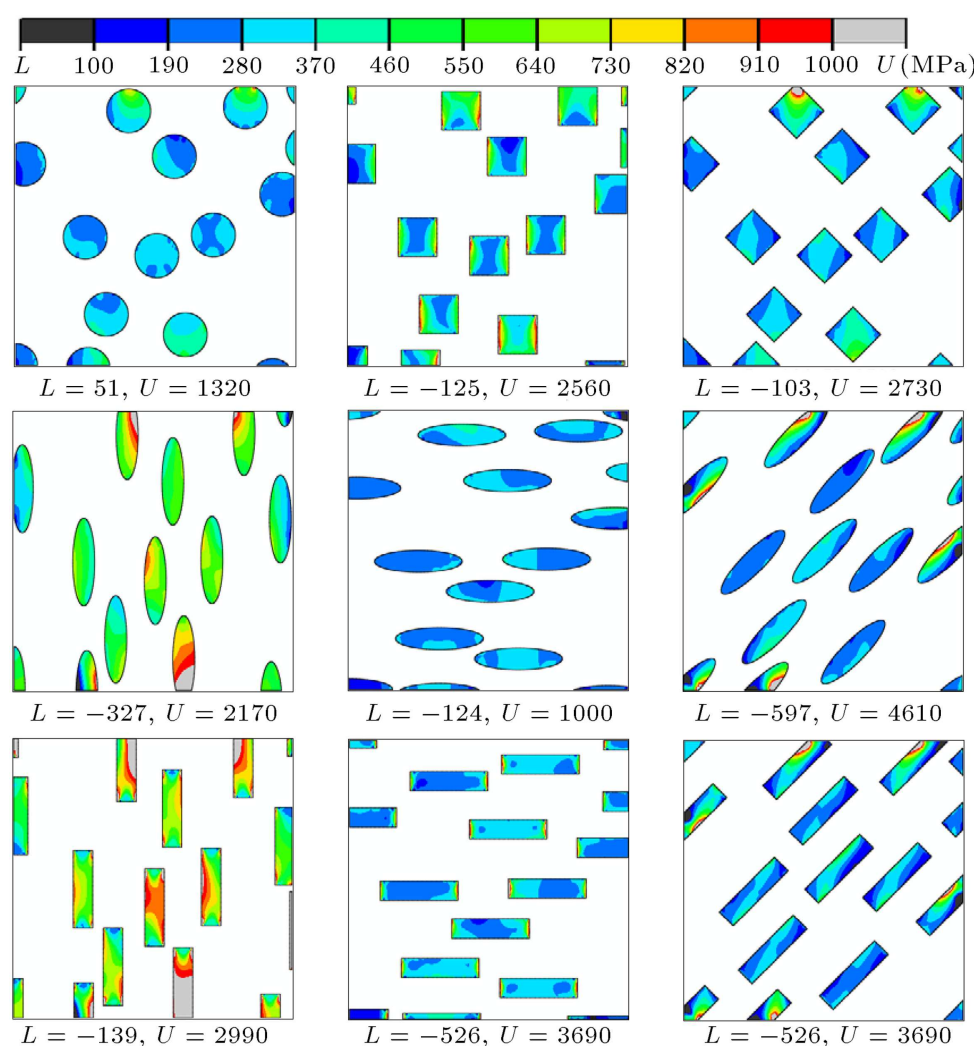

Figure 6. Contours of the maximum principle stress in the particles of size $1 \mu \mathrm{m}$ at the average axial strain $1 \%$ for the composites containing various particle shapes and orientations. 
compared to the circular particles, particles with sharp angles that are only horizontal/vertical have also higher maximum principle stress. However, for horizontal rectangular particles, the region with high maximum principle stress is confined to the small edges of the particles; for the rest of region of the particles, similar to the horizontal elliptical particles, the maximum principle stresses are in the order of those for the circular particles. For the inclined particles, the maximum principle stress is similar to the circular particles. This is consistent with the fact that the overall response of composites with inclined particles is similar to the composite with circular particles. Therefore, existence of sharp corners does not necessarily facilitate particle fracture and the orientation of the particle has significant effect on particle fracture. Since all these observations are more pronounced for small particle sizes, one can relate them to the dislocation strengthening mechanism, which is affected by the deformation inhomogeneity within the matrix, and in turn it influences stresses within the particles.

\subsection{Effects of particle size distribution}

Yan et al. [5] used the laser particle size analyzer to determine the size distribution of particles in their composites, such that the standard deviation for average particle sizes of $1,5,20$ and $56 \mu \mathrm{m}$ was reported in the range of $0.18 \sim 0.23$ (cf. Table 1 in [5]). Accordingly, here, we incorporate size distribution of $\mathrm{SiC}$ particles in our composite models so that the size of each particle in the model is selected from a Gaussian distribution with average $d$ and standard deviation of $0.21 d$, where $d=1$, 5,20 , and $56 \mu \mathrm{m}$. Corresponding stress-strain curves obtained for the composites with circular particles are shown in Figure 7 . It is seen that the curves are more or less the same as those in Figure 2, indicating that

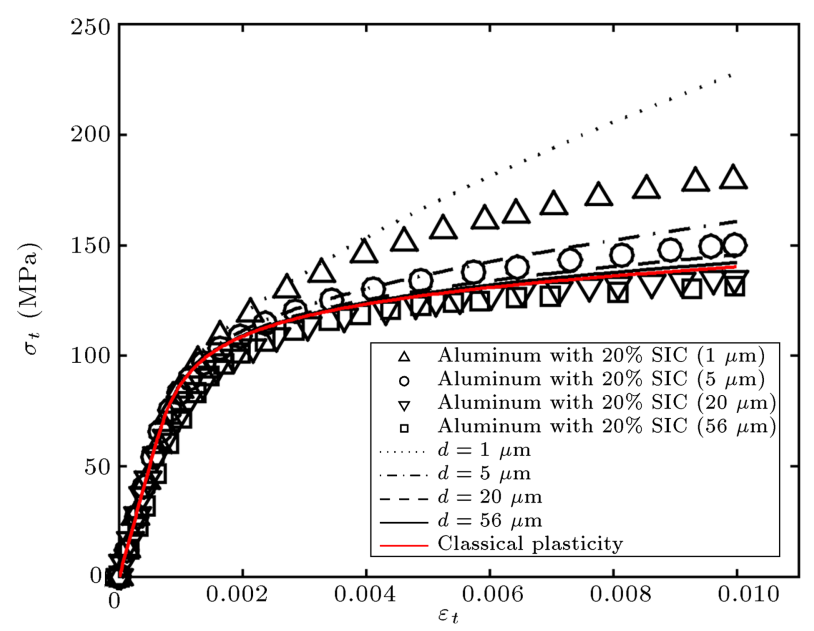

Figure 7. Predicted stress-strain curves of the composites with circular particles. A Gaussian distribution with average $d$ and standard deviation of $0.21 d$ is employed to determine particle sizes in each case.

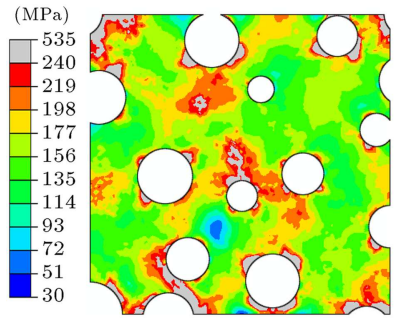

(a)

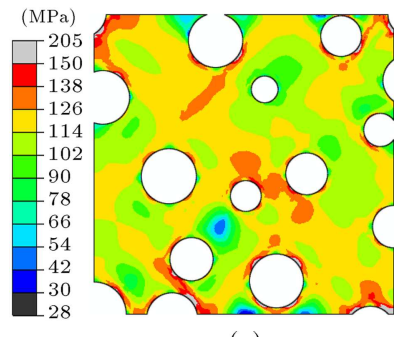

(c)

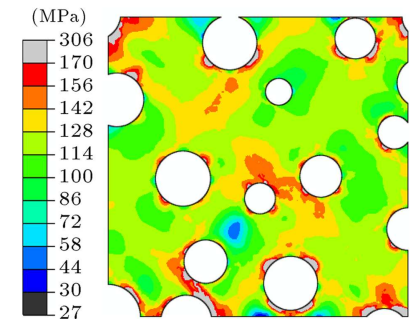

(b)

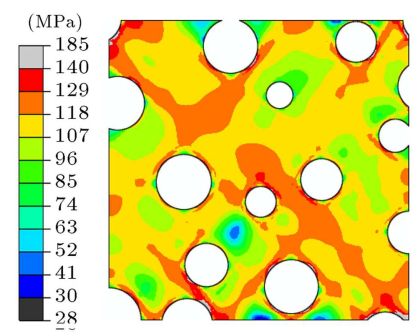

(d)
Figure 8. Contours of von Mises effective stress in the matrix at average axial strain $1 \%$ for the composites with average particle sizes of (a) d=1 $\mu \mathrm{m}$, (b) $\mathrm{d}=5 \mu \mathrm{m}$, (c) $\mathrm{d}=20 \mu \mathrm{m}$, and $(\mathrm{d}) \mathrm{d}=56 \mu \mathrm{m}$. A Gaussian distribution with average $d$ and standard deviation of $0.21 d$ is employed to determine particle sizes in each case.

the particle size distribution has very mild effect on composites' overall behavior.

Figure 8 shows the corresponding distribution of von Mises effective stress in the matrix at the average axial strain $1 \%$ of the composites. Comparing the results in this figure with those in Figure 3 reveals that stress inhomogeneity in the matrix is also mildly affected by the particle size distribution. It is noteworthy that other composites with different particle shape and orientation similarly showed low sensitivity to particle size distribution, which is not given here.

\section{Conclusions}

We used the Continuum theory of Mechanism-based Strain Gradient (CMSG) plasticity [13] to investigate particle size effects in metal matrix composites. A (two-dimensional) multi-particle unit cell model in plane-strain conditions, as in [5], was adopted. Predictions of particle size-dependent plastic behavior in the composites with various sizes of circular particles, i.e. $1,5,20$, and $56 \mu \mathrm{m}$, were presented and compared with the experimental data given in [5]. By decreasing particle size, the flow stress increases with high sensitivity to small particle size. A quantitative agreement between simulations and experiments was achieved at small plastic strains. However, due to the lack of incorporation of particle cracking and interfacial debonding in the present model, the hardening rate was somewhat overestimated. Analyses were carried out for 
four realizations of particle positions in the matrix, and very mild effects of particle positions were found.

As the next and main outcome of this contribution, effects of particle features, including shape, orientation, and size distribution, were considered. Analyses that carried out on the composites containing squared, rectangular, and elliptical (with aspect ratio four) particles of various orientations with respect to the loading direction (i.e., vertical, horizontal, and 45degree inclined) revealed that:

- The stress inhomogeneity in the matrix depends strongly on the shape and orientation of particles;

- The size effects predicted in CMSG depend on the shape and orientation of particles with quite high sensitivity to small particle size;

- The composites with inclined particles have the lowest flow stresses;

- The maximum principle stress-capable of predicting particle fracture-of particles with high aspect ratio that are vertical is substantially higher than that of the circular particle. Inclined particles with/without sharp angles have maximum principle stresses in the order of circular particles.

All these emphasize that the particle shape and orientation must be considered for designing particle reinforced metal matrix composites, especially, for small particle sizes, namely less than $5 \mu \mathrm{m}$. Finally, according to the particle size distributions of composites in [5], we modeled composites containing particles with average size $d$ and standard deviation of $0.21 d$. Very mild effect of particle size distribution on the behavior of composite was pronounced.

\section{References}

1. Lloyd, D.J. "Particle reinforced aluminum and magnesium matrix composites", International Materials Reviews, 39, pp. 1-23 (1994).

2. Ling, Z., Luo, L. and Dodd, B. "Experimental study on the formation of shear bands and effect of microstructure in $2124 \mathrm{Al} / \mathrm{SiCp}$ composite under dynamic compression", Journal de Physics, 4, pp. C8-453-458 (1994).

3. Kiser, M.T., Zok, F.W. and Wilkinson, D.S. "Plastic flow and fracture of a particulate metal matrix composite", Acta Materialia, 44, pp. 3465-3476 (1996).

4. Ganesh, V.V. and Chawla, N. "Effect of particle orientation anisotropy on the tensile behavior of metal matrix composites: experiments and microstructurebased simulation", Materials Science and Engineering A, 391, pp. 342-353 (2005).

5. Yan, Y.W., Geng, L. and Li, A.B. "Experimental and numerical studies of the effect of particle size on the deformation behavior of the metal matrix composites",
Materials Science and Engineering A, 448, pp. 315-325 (2007).

6. Su, Y., Ouyang, Q., Zhang, W., Li, Z., Guo, Q., Fan, G. and Zhang, D. "Composite structure modeling and mechanical behavior of particle reinforced metal matrix composites", Materials Science and Engineering A, 597, pp. 359-369 (2014).

7. Nan, C.W. and Clarke, D.R. "The influence of particle size and particle fracture on the elastic/plastic deformation of metal matrix composites", Acta Materialia, 44, pp. 3801-3811 (1996).

8. Dai, L.H., Ling, Z. and Bai, Y.L. "Size-dependent behavior of particle-reinforced metal-matrix composites", Composites Science and Technology, 61, pp. 1057-1063 (2001).

9. Xue, Z., Huang, Y. and Li, M. "Particle size effect in metallic materials: a study by the theory of mechanism-based strain gradient plasticity", Acta Materialia, 50, pp. 149-160 (2002).

10. Gao, H., Huang, Y., Nix, W.D. and Hutchinson, J.W. "Mechanism-based strain gradient plasticity. I. Theory", Journal of the Mechanics and Physics of Solids, 47, pp. 1239-1263 (1999).

11. Bao, G., Hutchinson, J.W. and McMeeking, R.M. "Particle reinforcement of ductile matrices against plastic flow and creep", Acta Metallurgica et Matererialia, 39, pp. 1871-1882 (1991).

12. Qu, S., Siegmund, T., Huang, Y., Wu, P.D., Zhang, F. and Hwang, K.C. "A study of particle size effect and interface fracture in aluminum alloy composite via an extended conventional theory of mechanismbased strain-gradient plasticity", Composites Science and Technology, 65, pp. 1244-1253 (2005).

13. Huang, Y., Qu, S., Hwang, K.C., Li, M. and Gao, H. "A conventional theory of mechanism-based strain gradient plasticity", International Journal of Plasticity, 20, pp. 753-782 (2004).

14. Shao, J.C., Xiao, B.L., Wang, Q.Z., Ma, Z.Y. and Yang, K. "An enhanced FEM model for particle size dependent flow strengthening and interface damage in particle reinforced metal matrix composites", Composites Science and Technology, 71, pp. 39-45 (2011).

15. Gao, H. and Huang, Y. "Taylor-based nonlocal theory of plasticity", International Journal of Solids and Structures, 38, pp. 2615-2637 (2001).

16. Taya, M., Lulay, K.E. and Lloyd, D.J. "Strengthening of a particulate metal matrix composite by quenching", Acta Metallurgica et Materialia, 39, pp. 73-87 (1991).

17. Fredriksson, P., Gudmundson, P. and Mikkelsen, L.P. "Finite element implementation and numerical issues of strain gradient plasticity with application to metal matrix composites", International Journal of Solids and Structures, 46, pp. 3977-3987 (2009).

18. Gudmundson, P. "A unified treatment of strain gradient plasticity", Journal of the Mechanics and Physics of Solids, 52, pp. 1379-1406 (2004). 
19. Shishvan, S.S. and Asghari, A.-H. "Particle size effect in metal matrix composites: a study by the continuum theory of stress gradient plasticity", Journal of Composite Materials, 50, pp. 1717-1723 (2016).

20. Chakravarthy, S.S. and Curtin, W.A. "Stress-gradient plasticity", Proceedings of National Academy of Science USA, 108, pp. 15716-15720 (2011).

21. Chakravarthy, S.S. and Curtin, W.A. "Stress gradient plasticity: concept and applications", Procedia IUTAM, 10, pp. 453-461 (2014).

22. Liu, D., He, Y., Zhang, B. and Shen, L. "A continuum theory of stress gradient plasticity based on the dislocation pile-up model", Acta Materialia, 80, pp. 350-364 (2014).

23. Qin, S., Chen, C., Zhang, G., Wang, W. and Wang, Z. "The effect of particle shape on ductility of SiCp reinforced 6061Al matrix composites", Materials Science and Engineering A, 272, pp. 363-370 (1999).

24. Chen, C.R., Qin, S.Y., Li, S.X. and Wen, J.L. "Finite element analysis about effects of particle morphology on mechanical response of composites", Materials Science and Engineering A, 278, pp. 96-105 (2000).

25. Taylor, G.I. "The mechanism of plastic deformation of crystals. Part I. Theoretical", Proceedings of the Royal Society A, 145, pp. 362-387 (1934).
26. Arsenlis, A. and Parks, D.M. "Crystallographic aspects of geometrically-necessary and statisticallystored dislocation density", Acta Materialia, 47, pp. 1597-1611 (1999).

27. Kok, S., Beaudoin, A.J. and Tortorelli, D.A. "A polycrystal plasticity model based on the mechanical threshold", International Journal of Plasticity, 18, pp. 715-741 (2002).

28. Hibbitt, D., Karlsson, B. and Sorensen, P., ABAQUS Analysis User's Manual Version 6.9, USA (2009).

\section{Biographies}

Siamak Soleymani Shishvan, $\mathrm{PhD}$, is an Assistant Professor in Faculty of Civil Engineering, University of Tabriz, Tabriz, Iran. His research interests include multi-scale modelling, plasticity, and fracture in materials.

Amir-Hossein Asghari received his MSc in Structural Engineering, in 2015, from University of Tabriz, Tabriz, Iran. His research interests include structural modellings. 